\title{
A Case Report on Yolk Sac Tumour in Pregnancy
}

\author{
Deepika Kapil ${ }^{1}$, Uday Mahajan ${ }^{2}$ \\ ${ }^{1}$ Departments of Obstetrics \& Gynaecology, and Medicine, Dr Rajendra Prasad Govt. Medical College, Kangra \\ at Tanda, Himachal Pradesh, India \\ ${ }^{2}$ Senior Resident, Department of Medicine, Dr Rajendra Prasad Govt. Medical College, Kangra at Tanda, \\ Himachal Pradesh, India \\ Corresponding Author: Uday Mahajan
}

\begin{abstract}
We present a case of 29-year-old female with chief complaint of amenorrhea since 2-months and pain abdomen for 15 days

There was a sudden worsening in the condition of patient as $\mathrm{Hb}$ of patient fell from $9.6 \mathrm{~g} / \mathrm{dl}$ to 6 $\mathrm{g} / \mathrm{dl}$ and ascites of the patient increased. Hence, the patient was decided for exploratory laparotomy after giving 2 blood transfusions. On opening the abdomen, there was about 3 liter of ascitic fluid which was hemorrhagic. Caking of omentum with dense adhesions of omentum with uterus and ascending and transverse colon was present. Grossly tumor was smooth surfaced with no superficial vessels over it. There were multiple papillary excrescences seen on the surface of tumor.

Left tube could not be visualized due to large ovarian mass right tube and ovary was normal. TAH with BSO with total omentectomy was done. Three units of fresh frozen plasma and three units of whole blood were given during surgery.

On histopathology examination, it was confirmed as yolk sac tumour

Conclusion: There should be further discussion regarding the management of advanced yolk sac tumours in pregnancy
\end{abstract}

Keywords: Yolk Sac Tumour, Pregnancy, Amenorrhea

\section{INTRODUCTION}

Yolk sac tumor, previously known as endodermal sinus tumor is a malignant tumor arising from germ cell of ovary, which shows differentiation into primitive endodermal structures. It constitutes 1-2\% of all ovarian malignancies and third most common type of ovarian malignancies. Before introduction of combination chemotherapy, mortality rate was very high, reaching up to $100 \%$ within three years of diagnosis. Introduction of vincristine, dactinomycin and cyclophosphamide regimen has increased the survival rate up to $94 \%{ }^{2}$. But, when this tumor is encountered in pregnancy, mortality and morbidity of both mother and baby increases due to technical, ethical and religious reasons.

\section{CASE REPORT}

We present a case of 29-year-old female with chief complaint of amenorrhea since 2-months and pain abdomen for 15 days. On examination, tenderness over periumblical area and distension was present. A hard mass of about 22-24-week size with restricted mobility and arising from pelvis fluid thrill was also present.

On ultrasound examination, there was single intrauterine gestational sac seen with solid cystic mass in pelvis with ascites.

On magnetic resonance imaging (MRI) examination, large multiseptated lesion with solid component with dense internal echoes and internal vascularity with gross ascites with omental thickening.

It was likely an ovarian neoplasm with peritoneal spread. Her tumour markers were LDH -139, CA-125 -58.8, AFP -0.86, CEA - 1.48. Ascitic tap was done and sent for malignant cytology; however, the report was negative. 
There was a sudden worsening in the condition of patient as $\mathrm{Hb}$ of patient fell from $9.6 \mathrm{~g} / \mathrm{dl}$ to $6 \mathrm{~g} / \mathrm{dl}$ and ascites of the patient increased. Hence, the patient was decided for exploratory laparotomy after giving 2 blood transfusions. On opening the abdomen, there was about 3 liter of ascitic fluid which was hemorrhagic. Caking of omentum with dense adhesions of omentum with uterus and ascending and transverse colon was present. Grossly tumor was smooth surfaced with no superficial vessels over it. There were multiple papillary excrescences seen on the surface of tumor.

Left tube could not be visualized due to large ovarian mass right tube and ovary was normal. TAH with BSO with total omentectomy was done. Three units of fresh frozen plasma and three units of whole blood were given during surgery.

On histopathology examination, it was confirmed as yolk sac tumour.

\section{DISCUSSION}

Yolk sac tumour, a malignant tumour arising from the germ cells of ovary are more commonly seen in children and young adults, median age being 19 years. These are rarely seen in women after menopause. The mode of presentation being abdominal distension, pain abdomen and sometimes mass per abdomen. ${ }^{1}$ Most of the times these tumors are detected late in pregnant women as the symptoms are obscured due to physiological changes of pregnancy. The diagnosis of yolk cell tumor during pregnancy requires high degree of suspicion. Increased serum tumor marker like alpha feto-protein, beta HCG and LDH will help in making diagnosis and assessing recurrence after treatment. ${ }^{5}$

Ultrasound and MRI are the radiological investigations available to diagnose and stage the tumour during pregnancy. ${ }^{2}$ Once diagnosed, treatment is challenging and depends upon multi factors like parity, duration of pregnancy, stage of tumor, general condition of patient; it is often possible to continue the pregnancy by debulking surgeries where tumor is resected completely. Since more than $50 \%$ of stage 1 tumor has micro-metastasis, tumor must be resected as much as possible. Prior to the availability of combination chemotherapy, mortality was very high even for stage 1 disease.

Introduction of combination chemotherapy with vincristine, dactinomycin and cyclophosphamide radically changed the mortality rate. Recently introduced protocol like Bleomycin, Etoposide and Cysplatin, have further increased the survival rate. Since, incidence of yolk sac tumour during pregnancy is very rare, no literature of properly conducted studies is available. ${ }^{3}$ Very few case reports could be found on internet where tumour was diagnosed in 3rd trimester and baby was delivered normally and tumour treated later. Luciano Souza Viana et al. published a case where yolk sac tumor was diagnosed in 20-year-old lady with 14 week pregnancy; pregnancy was preserved with 3 cycles of chemotherapy given with Cisplatin and Etoposide omitting Bleomycin. It is advisable to wait for a minimum of 21 days after chemotherapy to deliver a baby to avoid cytopenia in baby.

\section{CONCLUSION}

Yolk sac tumour is a rare tumor encountered in day today practice even rarer when associated with pregnancy. Management of such cases is a challenge for the surgeon due to limited diagnostic and therapeutic tools and lack of well-accepted protocol for management of such cases. Religious, ethical and economic factor should be considered along with thorough discussion with the patient and her relatives regarding the risks and hazards of the disease, investigations and the treatment options available.

Acknowledgement: None

Conflict of Interest: None

Source of Funding: None 
Declaration of Patient Consent:

Appropriate patient consent was taken prior to publication in the journal.

\section{REFERENCES}

1. Shimizu Y, Komiyama S, Kobayashi $\mathrm{T}$, Nakata K, Iida T. Successful management of endodermal sinus tumor of the ovary associated with pregnancy Department of Obstetrics and Gynecology, SaiseikaiUtsunomiya Hospital, Utsunomiya-shi, Tochigi, Japan.

2. Jung SE, Lee JM, Rha SE et-al. CT and MR imaging of ovarian tumors with emphasis on differential diagnosis. Radiographics. 22 (6): 1305-25. doi:10.1148/rg.226025033 Pubmed citation

3. Yamaoka T, Togashi K, Koyama T et-al. Yolk sac tumor of the ovary: radiologicpathologic correlation in four cases. $\mathbf{J}$ Comput Assist Tomogr. 24(4): 605-9. J
Comput Assist Tomogr (link) - Pubmed citation

4. Jeyakumar A, Chalas E, Hindenburg A. Sustained complete remission in a patient with platinum-resistant ovarian yolk sac tumor Oncology/Hematology Division, Department of Medicine, Winthrop University Hospital, Mineola, New York 11501, USA.

5. Nawa A, Obata N, Kikkawa F, Kawai M, Nagasaka T, Goto S, Nishimori K, Nakashima N. Prognostic factors of patients with yolk sac tumors of the ovary Department of Obstetrics and Gynecology, Nagoya University School of Medicine, Japan

How to cite this article: Kapil D, Mahajan U. A case report on yolk sac tumour in pregnancy. International Journal of Science \& Healthcare Research. 2021; 6(2): 286-288. DOI: https:// doi.org/10.52403/ijshr.20210451 